\title{
Accurate and Efficient Runtime Detection of Atomicity Errors in Concurrent Programs *
}

\author{
Technical Report DAR-05-26, Sept. 2005
}

\author{
Liqiang Wang \\ Computer Science Dept. \\ State University of New York at Stony Brook \\ liqiang@cs.sunysb.edu
}

\author{
Scott D. Stoller \\ Computer Science Dept. \\ State University of New York at Stony Brook \\ stoller@cs.sunysb.edu
}

\begin{abstract}
Atomicity is an important correctness condition for concurrent systems. Informally, atomicity is the property that every concurrent execution of a set of transactions is equivalent to some serial execution of the same transactions. In multi-threaded programs, executions of procedures (or methods) can be regarded as transactions. Correctness in the presence of concurrency often requires atomicity of these transactions. Tools that automatically detect atomicity violations can uncover subtle errors that are hard to find with traditional debugging and testing techniques.

This paper presents new algorithms for runtime (dynamic) detection of violations of conflict-atomicity and view-atomicity, which are analogous to conflict-serializability and view-serializability in database systems. In these algorithms, the recorded events are formed into a graph with edges representing the synchronization within each transaction and possible interactions between transactions. We give conditions on the graph that imply conflict-atomicity and view-atomicity. Experiments show that these new algorithms are more efficient in most experiments and are more accurate than previous algorithms with comparable asymptotic complexity.
\end{abstract}

Categories and Subject Descriptors D.2.5 [Software Engineering]: Testing and Debugging; D.2.4 [Software Engineering]: Software/Program Verification; D.1.3 [Programming Techniques]: Concurrent Programming

General Terms Reliability, Algorithms

Keywords concurrent programming, Java, atomicity, data race, serializability

\section{Introduction}

Multi-threading has become a common programming technique. Not only operating systems but also many applications are multi-

\footnotetext{
* This work was supported in part by NSF under Grant CCR-0205376 and CNS-0509230 and ONR under Grants N00014-02-1-0363 and N00014-041-0722.
}

[copyright notice will appear here] threaded. However, developing multi-threaded programs is difficult. Concurrency introduces the possibility of errors that do not exist in sequential programs. Furthermore, multi-threaded programs may behave differently from one run to another, because threads are scheduled indeterminately. For most systems, the number of possible schedules is enormous, and testing the system's behavior for each possible schedule is infeasible. Specialized techniques are needed to ensure that multi-threaded programs do not contain concurrency-related errors.

Threads often communicate by sharing data. Concurrent accesses to shared data should be properly synchronized. Two common errors are deadlocks and data races. Numerous static and dynamic (runtime) analysis techniques are designed to ensure that concurrent programs are free of deadlocks and data races. But this does not ensure the absence of all synchronization errors. Consider the implementation of Vector in Sun JDK 1.4.2, part of which appears in Figure 1. Consider the following execution of the program at the bottom of Figure 1: thread_1 constructs a new vector v2 from another vector $\mathrm{v} 1$ with $k$ elements by calling the constructor for Vector. But before the constructor completes, thread_1 yields execution to thread_2 immediately after statement 1 in the Vector constructor. thread_2 removes all elements of v1, and then thread_1 resumes execution at statement 2. The incorrect outcome is that v2 has $k$ elements, all of which are null, because the elementData array of v2 is allocated according to the previous size of v1. A more subtle error occurs if thread_2 executes v1.add(o) instead of v1.removeAllelements(). Then, if $k<10$, the length of elementData allocated in v2 is smaller than the new size of v1. Although a larger array is allocated in toArray to store the elements of $v 1$, the array is not returned to the constructor of v2, thus v2 will incorrectly be full of null elements. No exception is thrown in these scenarios. Methods size(), toArray(Object []), removeAllElements() and $\operatorname{add}(\mathrm{Object})$ are synchronized, hence there is no data race in these examples.

The incorrect behavior reflects a higher-level synchronization error, namely, lack of atomicity. Atomicity is well known in the context of transaction processing, where it is sometimes called serializability. The methods of concurrent programs are often intended to be atomic. A set of methods is atomic if concurrent invocations of the methods are always equivalent to performing the invocations serially (i.e., without interleaving) in some order. The first scenario of the example in Figure 1 contains two invocations, one of Vector(Collection) and one of removeAllElements(), which obviously do not have an equivalent serial execution. Therefore, these methods violate atomicity. Similarly, the second scenario also shows a violation of atomicity. 


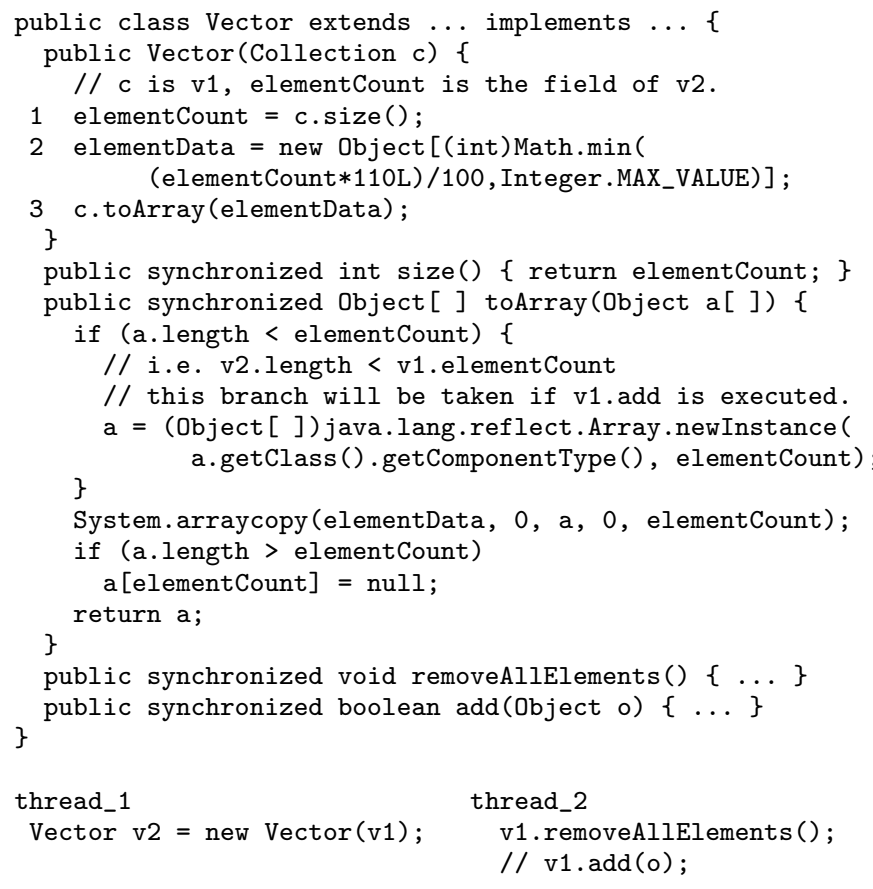

Figure 1. An example showing that the constructor of java.util. Vector in Sun JDK 1.4.2 violates atomicity.

Flanagan and Qadeer developed a type system for atomicity [8]. It can ensure that methods are atomic in all possible executions. However, type inference for the type system is NP-complete [6], so the type system may require manual annotation of the program.

In $[21,22]$, we proposed the reduction-based and block-based algorithms for runtime atomicity checking. Runtime analysis is less powerful than static analysis, because it cannot ensure correctness of all unexplored behaviors of the system, but may be more precise (i.e., give fewer false alarms) for the explored behaviors. Furthermore, runtime analysis does not require manual annotations of the code that are often required by type systems; this is a significant practical advantage.

This paper presents novel algorithms, called commit-node algorithms, for runtime checking of atomicity. The algorithms are offline, i.e., when the program terminates, they are applied to recorded information about the execution. The execution is partitioned into units. A unit is a sequence of events executed by a single thread. A transaction is a unit expected to behave atomically. For example, the sequence of events executed during a method invocation is often considered as a transaction. Our algorithms check whether every trace (i.e., interleaving) of these units is equivalent to a serial trace, where all events in each transaction of these units are consecutive. If so, we say that the transactions are atomic; if not, a potential atomicity violation is reported.

The monitor stores the events of each unit (including transactions) in a tree structure, called an access tree. Each node in an access tree denotes an access to an escaped variable (i.e., a variable accessible to multiple threads), or a synchronization operation (e.g., lock acquire and release). After the program terminates, the relationships between nodes in different trees are analyzed, and interedges are added between them to generate a forest. A node connected with inter-edges are called communication node. A communication node is called a commit node if none of its descendants are communication nodes. In a forest, if the access tree for each transaction has only one commit node, then the set of units is atomic.
By considering the synchronization, the commit-node algorithms do not merely look for violations of atomicity in the observed execution, but also attempt to determine whether the non-determinism of thread scheduling could allow violations in other executions.

This commit-node algorithms can check two kinds of atomicity, conflict-atomicity and view-atomicity, which are analogous to conflict-serializability and view-serializability in database systems.

Experiments show that these new algorithms are more efficient in most experiments and are more accurate than previous algorithms with comparable asymptotic complexity.

\section{Background}

This paper focuses on analyzing Java programs, but the techniques can be applied to other languages.

Event. Informally, an event is one step in an execution of a program. This paper considers the following operations on events: read and write escaped variables; acquire and release locks; start and join threads; start and exit invocations of methods; and the barrier synchronization operation discussed in Section 7.4. For example, synchronized $(l)\{$ body $\}$ in Java indicates two events (in addition to the events performed by the body): acquiring lock $l$ at the entry point and releasing it at the exit point. Two distinct accesses (even using the same operation) to a variable are different events. Let $h e l d(e)$ denote the locks held by the thread executing event $e$ when $e$ is executed.

Transaction Boundaries. Executions of the following code fragments are considered as transactions by default in this paper: nonprivate methods, synchronized private methods, and synchronized blocks inside non-synchronized private methods; as exceptions, the executions of the main () method in which the program starts and the executions of run ( ) methods of classes that implement Runnable are not considered as transactions, because these executions represent the entire executions of threads and are often not expected to be atomic. Moreover, start, join and barrier operations are treated as unit boundaries, i.e., they separate the preceding events and following events into different units, and are not contained in any unit. We adopt this heuristic because execution fragments containing these operations are typically not atomic and hence are not expected to be transactions. The events not in transactions form non-transactional units. All events in one non-transactional unit have the same thread period id (introduced in Section 7.3). Note that for nested transactions, we check atomicity of only the outermost transactions, since they contain the inner transactions.

Trace. A trace tr is a sequence of events. Given $\langle T, E\rangle$, where $T$ is a set of transactions, and $E$ is a set of non-transactional units, $a$ trace of $\langle T, E\rangle$ is an interleaving of events from units in $T \cup E$ that is consistent with the original order of events from each thread and with the synchronization events (e.g., no lock is held by multiple threads at the same time). A trace of $\langle T, E\rangle$ must contain all events from units in $T \cup E$ unless the trace ends in deadlock. This paper assumes that $E$ contains no synchronization; this assumption is satisfied if synchronized blocks are considered to be transactions.

Initial Read and Final Write. Let $e_{x}^{r}$ and $e_{x}^{w}$ denote a read event and a write event to variable $x$, respectively. $e_{x}^{w}$ is the writepredecessor of $e_{x}^{r}$ in a trace $t r$ if $e_{x}^{w}$ is the last write to $x$ that precedes $e_{x}^{r}$ in $t r . e_{x}^{r}$ is called a unit-initial read if $e_{x}^{r}$ does not have any write-predecessor in its own unit in all traces. $e_{x}^{r}$ is called a trace-initial read in trace $t r$ if $e_{x}^{r}$ is not preceded by a write to $x$ in $t r$. Its write-predecessor is defined to be an imaginary write event $e_{x}^{\text {init }}$ at the beginning of the trace. A write event $e_{x}^{w}$ is called a unit-final write if it is the last write to $x$ in its unit; a write event $e_{x}^{w}$ is called a trace-final write in a trace if it is the last write to $x$ in the trace. 
Conflict-Equivalence. Two traces $t r_{1}$ and $t r_{2}$ for $\langle T, E\rangle$ are conflict-equivalent iff (i) they contain the same events, and (ii) for each pair of conflicting events, the two events appear in the same order in both traces. This corresponds to conflict equivalence in transaction processing in database systems [3].

View-Equivalence. Two traces $t r_{1}$ and $t r_{2}$ for $\langle T, E\rangle$ are viewequivalent iff ( $i$ ) they contain the same events, (ii) each read event has the same write-predecessor in both traces, and (iii) each variable has the same trace-final write event in both traces. This corresponds to view equivalence in transaction processing [3]. It is easy to show that conflict-equivalence implies view-equivalence [3]. But the converse does not hold.

Conflict-Serializability and View-Serializability. A trace of $\langle T, E\rangle$ is serial if the events of each transaction of $T$ form a contiguous subsequence of the trace. Note that the events in each nontransactional unit of $E$ are not required to be contiguous. A trace of $\langle T, E\rangle$ is conflict-serializable if it is conflict-equivalent to some serial trace of $\langle T, E\rangle$. A trace of $\langle T, E\rangle$ is view-serializable if it is view-equivalent to some serial trace of $\langle T, E\rangle$. Conflictserializability of a trace $t r$ for $\langle T, E\rangle$ can be decided in polynomial time [3]. Let $g$ be the serialization graph for $t r$, which is a directed graph whose nodes are the units of $T \cup E$, and which contains an edge from node $t_{i}$ to node $t_{j}$ if $i \neq j$ and some event of $t_{i}$ precedes a conflicting event of $t_{j}$ in $t r . t r$ is conflict-serializable iff $g$ does not contain any cycle containing two or more transactions. In contrast, checking view serializability is NP-complete [16].

Conflict-Atomicity and View-Atomicity. $\quad\langle T, E\rangle$ is conflict-atomic if every trace of $\langle T, E\rangle$ is conflict-serializable. $\langle T, E\rangle$ is viewatomic if every trace of $\langle T, E\rangle$ is view-serializable. It is easy to show that conflict-atomicity implies view-atomicity, but the converse does not hold. As an example, consider $\left\langle\left\{t_{1}, t_{2}\right\}, \emptyset\right\rangle$, where $t_{1}$ is $W_{1}(x) W_{2}(x)$, and $t_{2}$ is $W(x)$. When $t_{2} . W(x)$ happens between $t_{1} \cdot W_{1}(x)$ and $t_{1} \cdot W_{2}(x)$, the trace does not have any conflict-equivalent serial trace, hence $\left\langle\left\{t_{1}, t_{2}\right\}, \emptyset\right\rangle$ is not conflictatomic; but the trace is view-equivalent to a serial trace $t_{2} . W(x)$ $t_{1} . W_{1}(x) t_{1} \cdot W_{2}(x)$, and all the other possible traces are serial, hence $\left\langle\left\{t_{1}, t_{2}\right\}, \emptyset\right\rangle$ is view-atomic.

Potential for Deadlock. $\quad\langle T, E\rangle$ has potential for deadlock if some trace of $\langle T, E\rangle$ ends in deadlock. A trace that ends in deadlock with some thread in the middle of a transaction is not equivalent to any serial trace. Therefore, this paper assumes that $\langle T, E\rangle$ has no potential for deadlock. This can be checked using the goodlock algorithm [10] or an extension of it [2].

\section{Access Forest and Commit-Node Reduction}

\subsection{Access Tree}

During execution of the instrumented program, the monitor records all events for each unit into an access tree. In such a tree, each leaf node is called an access node and denotes an access to an escaped variable. Each non-leaf node except for the root is called a synchronization node and denotes a synchronization block. The root node denotes the whole unit. The local orders of events within a unit are denoted by the order of branches in the tree. An example appears in Figure 2, where $R(v)$ and $W(v)$ ( $v$ is $x$ or $y$ ) denote a read event and a write event to $v$, respectively; $a c q(l)$ and $\operatorname{rel}(l)$ denote an acquire and a release of lock $l$, respectively. Since each node in an access tree denotes a set of events, a node and the set of events it denotes are used interchangeably in our description.

\subsection{Access Forest}

An access forest consists of a set of access trees and edges called inter-edges between access trees from concurrent units. Section

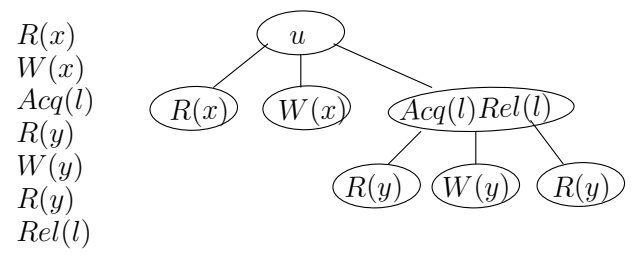

Figure 2. The access tree for a unit $u$. All events are shown on the left; time increases from top to bottom.

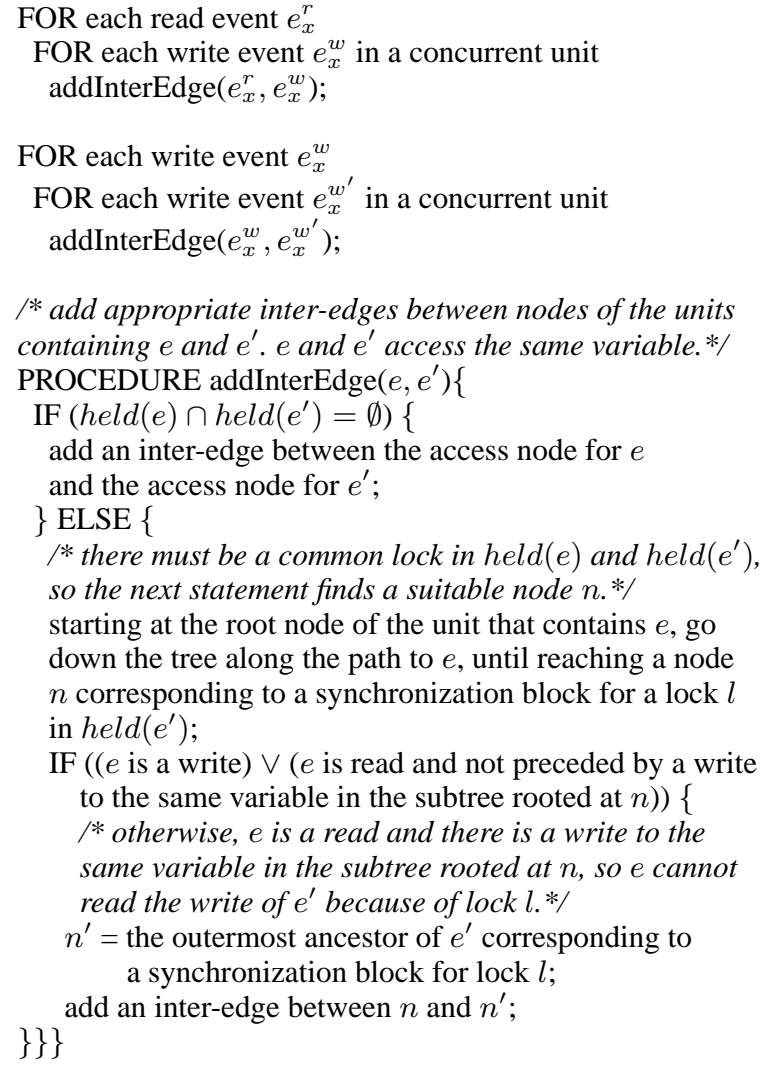

Figure 3. The algorithm to add inter-edges for an arbitrary escaped variable $x$ in the conflict-forest.

7.4 describes a happen-before analysis to determine whether two units are concurrent. The edges inside each tree are called treeedges. Nodes with an incident inter-edge are called communication nodes; they denote a potential interactions between the corresponding units. Checking conflict-atomicity and view-atomicity require different inter-edges. The access forest used for checking conflictatomicity is called conflict-forest; the access forest used for checking view-atomicity is called view-forest.

\subsubsection{Conflict-Forest}

In the conflict-forest, there are two kinds of relationships denoted by inter-edges between two concurrent units. The first kind of relationship is between a node associated with a write in one of the units and a concurrent node associated with a read to the same variable in the other unit, if the read can read the written value by the write in some trace. The second kind of relationship connects two concurrent nodes associated with two writes to the same variable in the two. 


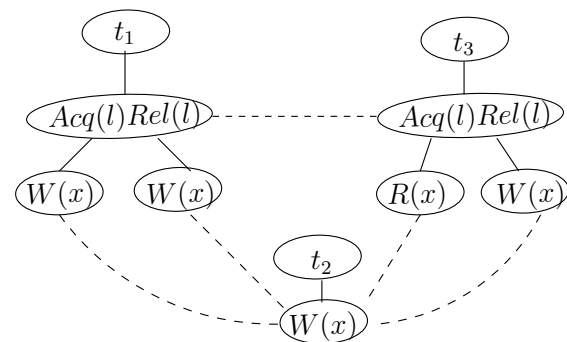

Figure 4. A conflict-forest. The inter-edges are shown as dotted lines.

We say "associated with" above because the inter-edge is not necessarily added directly between the access nodes representing those two accesses. Instead, for each pair of accesses satisfying the above conditions, if there is at least one lock that is held when both operations are performed, then we find the outermost of those common locks, and add an inter-edge between the corresponding synchronization nodes, because this is the granularity at which the parts of the units containing those accesses can be interleaved; if no such lock exists, then an inter-edge is added directly between the access nodes representing those two accesses. By assumption, the set of units does not have potential for deadlock, so the notion of outermost common lock for two accesses is well defined; if there is potential for deadlock, the threads that execute the two accesses could acquire two locks in different orders without first acquiring a common lock.

Intuitively, $\langle T, E\rangle$ is conflict-atomic, if in all traces of $\langle T, E\rangle$, the events of each transaction of $T$ can be repeatedly swapped with adjacent events without affecting the rest of the trace, until the trace is serial, i.e., the events of each transaction are contiguous. If two nodes are connected by an inter-edge, they cannot be swapped. Thus, a node with incident inter-edges is like a non-mover in Lipton's reduction $[15,5,21,22]$.

Figure 3 shows the algorithm to add inter-edges. Figure 4 shows the conflict forest for a set of three units. Note that an inter-edge can denote multiple relationships of the kinds described above. For example, the inter-edge between $t_{1}$ and $t_{3}$ in Figure 4 denotes two relationships: one is that $t_{3} \cdot R(x)$ can read the value written by $t_{1} . W(x)$, and the other is between $t_{1} . W(x)$ and $t_{3} . W(x)$.

Besides checking atomicity, the conflict-forest can also be used for detecting data races, since each access node with incident interedges indicates a data race.

\subsubsection{View-Forest}

The view-forest has three kinds of relationships between two concurrent units $u_{1}$ and $u_{2}$ denoted by inter-edges. (1) The first kind of relationship is between a node of $u_{1}$ associated with a write and a node of $u_{2}$ associated with a read, if the read can read the written value by the write in some trace. (2) The second kind of relationship connects two nodes associated with two writes to the same variable, respectively, if both writes can be the write-predecessor of the same read in some traces. (3) The third kind of relationship connects two nodes associated with unit-final writes to the same variable.

The algorithm of adding inter-edges for view-forest is shown in Figure 5. It is similar to the algorithm in Figure 3. When adding an inter-edge between a read and its potential write-predecessor, we also add inter-edges between its all potential write-predecessors. $S(e)$ caches all potential write-predecessors for the read $e$ so far. Besides connecting this kind of writes, we also add inter-edges between the unit-final writes to the same variables, instead of adding

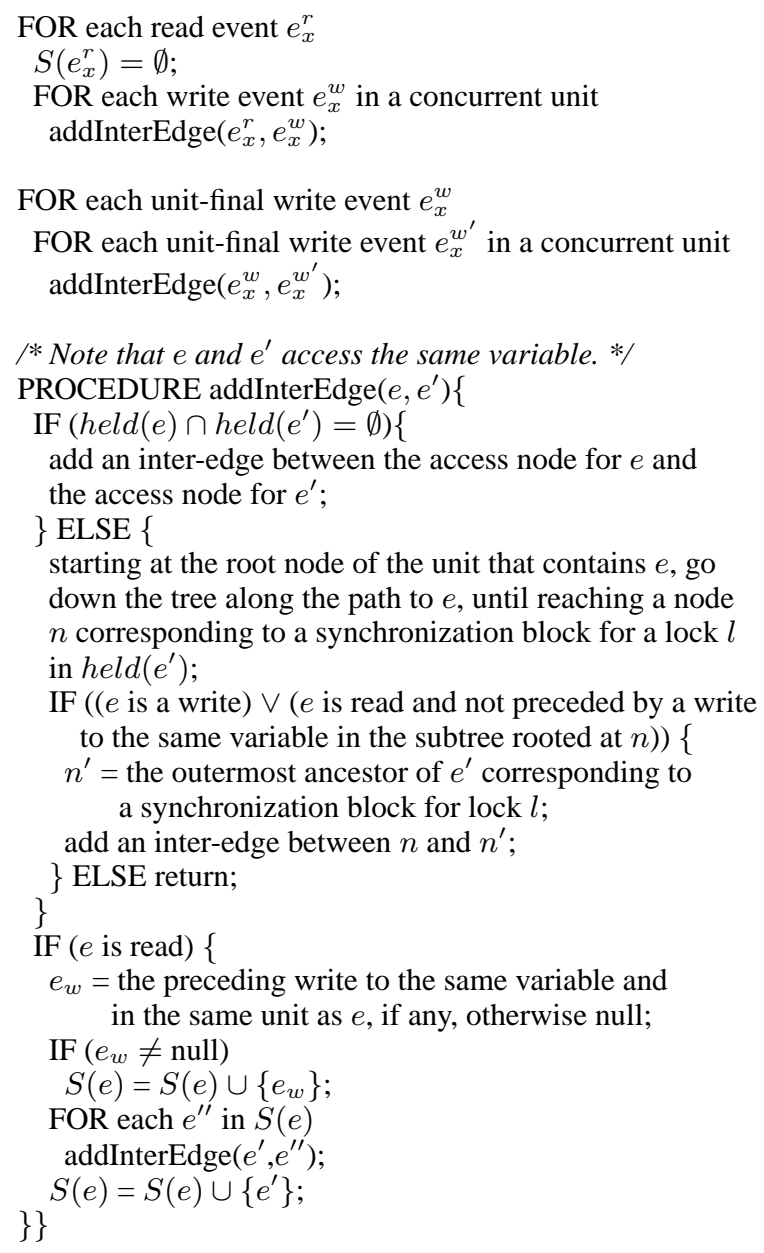

Figure 5. The algorithm to add inter-edges for an arbitrary escaped variable $x$ in the view-forest.

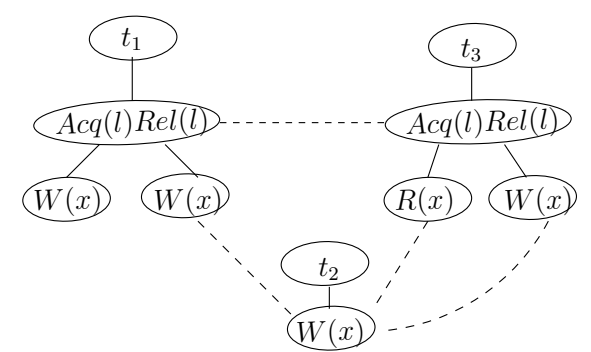

Figure 6. A view-forest. The inter-edges are shown as dotted lines.

inter-edges between every two writes to the same variables in conflict forest.

Figure 6 shows the view forest after applying the algorithm to the same three units in Figure 4.

\subsection{Commit-Node Reduction}

Let $n>n^{\prime}$ denote that a communication node $n$ contains (i.e., is an ancestor of) another communication node $n^{\prime}$. A communication node is called a commit node if it is not contained in any other communication nodes. For example, in Figure 6, the communication node " $A c q(l) \operatorname{Rel}(l)$ " in $t_{1}$ contains the communication node 
" $W(x)$ " which is a commit node since it does not contain any other communication nodes.

Intuitively, for a set of nodes $n_{1}>\ldots>n_{k}>n_{c}$, the commit node $n_{c}$ denotes a non-mover, $n_{i}(1 \leqslant i \leqslant k)$ denotes a larger nonmover which contains $n_{c}$. All events of $n_{1}$ (which also contains all events of $n_{2}, \ldots, n_{c}$ ) can be moved to the commit node position through swapping without affecting the other units in all traces. Thus, a transaction with at most one commit node is atomic, but a transaction with two or more commit nodes might be non-atomic. This is described formally in Section 4.

\section{The Commit-Node Algorithms for Checking Atomicity}

This section presents algorithms for checking conflict-atomicity and view-atomicity.

\subsection{Conflict-Atomicity}

Theorem 4.1. Suppose $\langle T, E\rangle$ has no potential for deadlock, and $E$ does not contain any synchronization operations. If each transaction of $T$ has at most one commit node in the conflict-forest, then $\langle T, E\rangle$ is conflict-atomic.

Proof. To prove that $\langle T, E\rangle$ is conflict-atomic, we need to show that there is a conflict-equivalent serial trace $t r^{\prime}$ for an arbitrary trace $\operatorname{tr}$ of $\langle T, E\rangle$. The general idea is to find a location of some event inside the commit node for each transaction, such that when all events of each transaction are moved to that location, the resulting trace is conflict-equivalent to the original trace. That location is called a commit point.

For a commit node $n$, if $n$ is an access node, its commit point is the location of the access event at $t r$; if $n$ is a synchronization node, its commit point is any arbitrary location inside the commit node at $t r$. According to the assumption in the theorem, each communication node contains only one commit node. $t r^{\prime}$ is constructed from $t r$ as follows: all events of the communication nodes that contain the commit node are moved to the commit point; all events of each transaction not in any communication node are also moved to the commit point of the transaction; all other events are not moved. $t r^{\prime}$ is serial because every transaction has only one commit node. In the following, we prove that $t r^{\prime}$ is a legal trace and is conflictequivalent to $t r$.

First, we observe that $t r^{\prime}$ is consistent with the synchronization events. This holds because $t r^{\prime}$ is serial, and $E$ does not contain any synchronization. So $t r^{\prime}$ is a trace for $\langle T, E\rangle$.

Next, we show that $t r^{\prime}$ is conflict-equivalent to $t r$. Consider conflicting events $e_{1}$ and $e_{2}$, where $e_{1}$ and $e_{2}$ occur in units $u_{1}$ and $u_{2}$ in $T \cup E$, respectively. Without loss of generality, suppose $e_{1}$ precedes $e_{2}$ in $t r$. Because $e_{1}$ and $e_{2}$ conflict, $u_{1}$ must contain a communication node $n_{1}$ containing $e_{1}$, and $u_{2}$ must contain a communication node $n_{2}$ containing $e_{2}$, and $n_{1}$ precedes $n_{2}$ in $t r$. After moving $e_{1}$ and $e_{2}$ to the commit points of $u_{1}$ and $u_{2}$, respectively, $e_{1}$ and $e_{2}$ appear at the same order in $t r$ and $t r^{\prime}$. Therefore, $t r$ is conflict-equivalent to $t r^{\prime}$.

The condition in Theorem 4.1 for conflict-atomicity is sufficient but not necessary. In Figure 7, the set of transactions is conflictatomic, even though $t_{1}$ contains multiple commit nodes. The following theorem shows that the condition in Theorem 4.1 is an exact test for conflict-atomicity of two transactions.

Theorem 4.2. Suppose $\langle T, \emptyset\rangle$ has no potential for deadlock, and $T$ contains only two transactions. $\langle T, \emptyset\rangle$ is conflict-atomic iff each transaction in Thas at most one commit node in the conflict-forest.

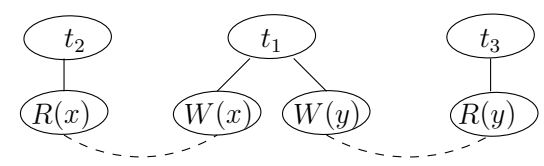

Figure 7. $\left\langle\left\{t_{1}, t_{2}, t_{3}\right\}, \emptyset\right\rangle$ is both conflict-atomic and view-atomic, but $t_{1}$ contains two commit nodes.

Proof. A proof sketch appears here; more details are in the Appendix.

" $\Leftarrow "$ : This direction follows from Theorem 4.1.

" $\Rightarrow$ ": Suppose $T=\left\{t, t^{\prime}\right\}$. We show that $\langle T, \emptyset\rangle$ is not conflictatomic if at least one transaction in $T$ has two or more commit nodes. Without loss of generality, suppose $t$ has at least two commit nodes. Let $n_{1}$ and $n_{2}$ denote two commit nodes of $t . t^{\prime}$ has at least one commit node. There must be a pair of conflicting events, denoted $e_{1}$ and $e_{1}^{\prime}$, with $e_{1} \in n_{1}$ and $e_{1}^{\prime} \in n_{1}^{\prime}$, where $n_{1}^{\prime}$ is a communication node in $t^{\prime}$, and there is an inter-edge between $n_{1}$ and $n_{1}^{\prime}$. Similarly, there must be another pair of conflicting events, denoted $e_{2}$ and $e_{2}^{\prime}$, with $e_{2} \in n_{2}$ and $e_{2}^{\prime} \in n_{2}^{\prime}$, where $n_{2}^{\prime}$ is a communication node in $t^{\prime}$, and there is an inter-edge between $n_{2}$ and $n_{2}^{\prime}$.

If one of $n_{1}^{\prime}$ and $n_{2}^{\prime}$ contains the other or if $n_{1}^{\prime}=n_{2}^{\prime}$, we can show that there is a trace $t r$ where $n_{1}^{\prime}$ and $n_{2}^{\prime}$ happen between $n_{1}$ and $n_{2}$. Otherwise, we can show that there is a trace $t r$ where $n_{1}^{\prime}$ and $n_{2}^{\prime}$ happen between $n_{1}$ and $n_{2}$, or $n_{1}$ and $n_{2}$ happen between $n_{1}^{\prime}$ and $n_{2}^{\prime}$. Hence, $t r$ does not have any conflict-equivalent serial trace.

For example, in Figure 4, $\left\langle\left\{t_{1}, t_{2}\right\}, \emptyset\right\rangle$ is not conflict-atomic according to Theorem 4.2 because $t_{1}$ contains two commit nodes when ignoring $t_{3}$. Similarly, $\left\langle\left\{t_{2}, t_{3}\right\}, \emptyset\right\rangle$ is not conflict-atomic, either.

The following theorem gives a more sophisticated (compared to Theorem 4.1) condition to decide conflict-atomicity (for any number of transactions). This theorem (unlike Theorem 4.1) is accurate enough to show that the set of transactions in Figure 7 is conflict-atomic. Note that, when considering cycles in the conflictforest, tree edges are treated as undirected edges.

Theorem 4.3. Suppose $\langle T, E\rangle$ has no potential for deadlock, and $E$ does not contain any synchronization operations. If all pairs (if they exist) of communication nodes from the same transaction that do not contain each other are not involved in any cycle of the conflict-forest, then $\langle T, E\rangle$ is conflict-atomic.

Proof. We prove the contrapositive. Suppose $\langle T, E\rangle$ is not conflictatomic. Thus, there is a trace $\operatorname{tr}$ for $\langle T, \emptyset\rangle$ that does not have any conflict-equivalent serial trace, and there is a directed cycle $c$ in the serialization graph for $t r$. Suppose that $c$ consists of $\left\langle t_{1}, t_{2}, \ldots, t_{n}\right\rangle$ in order, where $t_{1}, t_{2}, \ldots, t_{n} \in T$. $c$ implies that there must be an event $e_{1}$ of $t_{1}$ that happens before an event $e_{2}$ of $t_{2}$, an event $e_{2}^{\prime}$ of $t_{2}$ that happens before an event $e_{3}$ of $t_{3}, \ldots$, and an event $e_{n}^{\prime}$ of $t_{n}$ that happens before $e_{1}^{\prime}$ of $t_{1}$ in $t r$, where $e_{1}$ and $e_{2}$ access the same variable, $e_{2}^{\prime}$ and $e_{3}$ access the same variable, ...., and $e_{n}^{\prime}$ and $e_{1}^{\prime}$ access the same variable. This implies that there is a cycle $c^{\prime}$ in the conflict-forest. If $e_{i}$ and $e_{i}^{\prime}$ of $t_{i}$ for $i=1 . . n$ are in the same communication node, then both $e_{i}$ and $e_{i}^{\prime}$ happen before $e_{i+1}$ and $e_{i+1}^{\prime}$ in $t r$, for $i=1 . . n-1$. This contradicts the assumption that $e_{n}^{\prime}$ happens before $e_{1}^{\prime}$. Hence, there must be a transaction in $\left\{t_{1}, t_{2}, \ldots, t_{n}\right\}$ that has at least two communication nodes on $c^{\prime}$ that do not contain each other.

The commit-node algorithm for checking conflict-atomicity works as follows. (1) Instrument the source code of program to be 
tested as discussed in Section 7.1. (2) Execute the instrumented program, and dynamically construct the conflict-trees. (3) Add inter-edges after the execution terminates. (4) Check the conditions of Theorem 4.1; if they are satisfied, report that conflict-atomicity holds; otherwise, check the conditions of Theorem 4.3, then report conflict-atomicity holds or not according to whether the conditions are satisfied.

Although this algorithm may report false alarms since the conditions in Theorem 4.1 and Theorem 4.3 are sufficient but not necessary. But we believe that this happens very rarely. In the experiments of Section 8, all the warnings for non-conflict-atomicity reported by the algorithm are confirmed to be true by Theorem 4.2.

Let $|T|, n_{t}$, and $n_{e}$ denote the number of transactions, the maximum number of events in a transaction, and the number of events in the whole execution (including non-transactional units), respectively. Theorem 4.3 requires checking, for each pair of communication nodes of the same transaction, whether they are involved in a cycle, i.e., whether each of them is reachable from the other. There are $O\left(|T| \times n_{t}^{2}\right)$ such pairs, and checking whether two nodes are reachable from each other takes time $O\left(n_{e}^{2}\right)$, so the worst-case time complexity of the algorithm is $O\left(|T| \times n_{t}^{2} \times n_{e}^{2}\right)$.

\subsection{View-Atomicity}

Theorem 4.4. Suppose $\langle T, E\rangle$ has no potential for deadlock, and $E$ does not contain any synchronization operations. If each transaction of $T$ has at most one commit node in the view-forest, then $\langle T, E\rangle$ is view-atomic.

Proof. A proof sketch appears here; more details are in the Appendix. For any trace $\operatorname{tr}$ of $\langle T, E\rangle$, we prove that it has a viewequivalent serial trace $t r^{\prime}$, which is constructed in the same way as in the proof of Theorem 4.1. The definition for the commit point of each transaction is the same as there. By the same reasoning as in that proof, $t r^{\prime}$ is serial and consistent with the synchronization operations, so $t r^{\prime}$ is a trace for $\langle T, E\rangle$. Next we prove in two steps that $t r^{\prime}$ is view-equivalent to $t r$. (1) We first prove that each read has the same write-predecessor in $t r$ and $t r^{\prime}$. The main observation is: if two communication nodes are connected by an inter-edge, then the sets of nodes contained in them cannot be interleaved with each other in any trace. Because there are inter-edges between communication nodes associated with potential write-predecessors for the same read $e^{r}$, if one communication node $n^{w}$ contains the actual write-predecessor of the read $e^{r}$ in $t r$, all the communication nodes associated with other potential write-predecessors for $e^{r}$ must happen in $t r$ before the events represented by $n^{w}$ or after the commit node associated with $e^{r}$. Hence, after moving all events in each transaction to its commit node, $e^{r}$ has the same write-predecessor in $t r^{\prime}$ and $t r$. (2) A similar proof shows that $t r$ and $t r^{\prime}$ have the same trace-final writes.

For example, in Figure $6,\left\langle\left\{t_{1}, t_{2}\right\}, \emptyset\right\rangle$ is view-atomic because each of $t_{1}$ and $t_{2}$ contains only one commit node in the viewforest. Note that $\left\langle\left\{t_{1}, t_{2}\right\}, \emptyset\right\rangle$ is not conflict-atomic since $t_{1}$ has two commit nodes in the conflict-forest.

The condition in Theorem 4.4 for view-atomicity is sufficient but not necessary. In the example of Figure $7,\left\langle\left\{t_{1}, t_{2}, t_{3}\right\}, \emptyset\right\rangle$ is view-atomic but $t_{1}$ contains multiple commit nodes. The following theorem shows that the condition in Theorem 4.4 is an exact test for view-atomicity of two transactions.

Theorem 4.5. Suppose $T$ has no potential for deadlock, and $T$ contains only two transactions. $\langle T, \emptyset\rangle$ is view-atomic iff each transaction in $T$ has at most one commit node in the view-forest.

Proof. " $\Leftarrow$ ": This implication is justified directly based on Theorem 4.4 .
" $\Rightarrow$ ": We prove the contrapositive, i.e., if at least one of the transactions has at least two commit nodes, then $\langle T, \emptyset\rangle$ is not viewatomic. According to the definition of view-forest, there are three kinds of inter-edge. (1) The first kind of inter-edge denotes the relationship between a read $e^{r}$ in a transaction $t$ and its potential write-predecessor $e^{w}$ in the other transaction. If the read has a preceding write $e_{p r e}^{w}$ in its own transaction, then a violation of view-atomicity is possible (because $e^{w}$ can occur between $e_{p r e}^{w}$ and $e^{r}$ by the definition of potential write-predecessor), so the desired implication holds. If the read does not have any preceding write in its own transaction, the read and its potential write-predecessor in the other transaction act like a pair of conflicting events, in the sense that their order in a trace determines that the two transactions must follow the order in all serial traces view-equivalent to the trace (this is true with two transactions, although it is not true with more transactions). This is the same property of inter-edges in the conflict-forest that is used in the proof of Theorem 4.2. (2) The second kind of inter-edge denotes the relationship between two writes that are potential write-predecessor for the same read; this indicates a violation of view-atomicity (because either there is a write to some variable $x$ in $t^{\prime}$ that can occur between a write to $x$ and a read to $x$ in $t$, or there is a read to $x$ in $t^{\prime}$ that can occur between two writes to $x$ in $t$ ), so the desired implication holds. (3) The third kind of inter-edge denotes the relationship between unitfinal writes of each transaction. With two transactions, these final writes also act like conflicting events, in the sense described above, so these edges have the same property as inter-edges in the conflictforest.

Thus, depending on the kind of edges present, either we immediately conclude that the desired implication holds, or all of the edges have the property of inter-edges in the conflict-forest used in the proof of $(\Rightarrow)$ in Theorem 4.2, and the rest of this proof is similar to that proof.

For example, in Figure $6,\left\langle\left\{t_{2}, t_{3}\right\}, \emptyset\right\rangle$ is not view-atomic because $t_{3}$ contains two commit nodes in the view-forest for $t_{2}$ and $t_{3}$. The following theorem gives a more sophisticated condition to check view-atomicity.

Theorem 4.6. Suppose $\langle T, E\rangle$ has no potential for deadlock, and $E$ does not contain any synchronization operations. If all pairs of communication nodes from the same transaction that do not contain each other are not involved in any cycle of the view-forest, then $\langle T, E\rangle$ is view-atomic.

Proof. The proof is similar to the proof of Theorem 4.3.

The commit-node algorithm for checking view-atomicity is similar to the algorithm for checking conflict-atomicity proposed in Section 4.1, except that the view-forest is constructed and checked based on Theorems 4.4 and 4.6. Similarly as before, the worst-case time complexity of the algorithm is $O\left(|T| \times n_{t}^{2} \times n_{e}^{2}\right)$.

\section{The Polynomial Equivalence of Conflict- and View-Atomicity}

The following theorem shows that the problems of checking conflict-atomicity and checking view-atomicity are polynomially reducible to each other. This result is somewhat surprising, considering that checking conflict serializability is in P [3] and checking view serializability is NP-complete [16], so they are not polynomially reducible unless $\mathrm{P}=\mathrm{NP}$. To simply the problem, we consider only transactions i.e., assume $E=\emptyset$; we expect that the result also holds without this restriction.

Theorem 5.1. The problems of checking conflict-atomicity and checking view-atomicity are polynomially reducible to each other 
when restricted to problem instances where the set $E$ of nontransactional units is empty.

Proof. A proof sketch appears here; more details are in the Appendix.

1. We first prove that the problem of checking conflict-atomicity is polynomially reducible to the problem of checking view-atomicity. We transform $T$ as follows. Let $l$ be a lock not used in $T$. For each variable $x$, each read $e_{x}^{r}$ is replaced by $e_{l}^{a c q} e_{x}^{r} e_{l}^{r e l}$; and each write $e_{x}^{w}$ is replaced by $e_{l}^{a c q} e_{x}^{r} e_{x}^{w} e_{l}^{r e l}$, where $e_{l}^{a c q}$ and $e_{l}^{r e l}$ represent an acquire and a release of $l$, respectively. Let $T^{\prime}$ denote the resulting set of transactions.

We prove that $T$ is conflict-atomic iff $T^{\prime}$ is view-atomic. " $\Rightarrow$ ": Every trace $t r^{\prime}$ of $T^{\prime}$ corresponds to some trace $t r$ of $T$. Every trace $t r$ of $T$ has a conflict-equivalent serial trace $t r_{s}$. We transform $t r$ and $t r_{s}$ in the same manner that is used to transform $T$, yielding traces $t r^{\prime}$ and $t r_{s}^{\prime}$ of $T^{\prime}$, respectively. We show in the Appendix that $t r^{\prime}$ is view-equivalent to the serial trace $t r_{s}^{\prime}$. "Æ": For each trace $t r^{\prime}$ of $T^{\prime}$ and its view-equivalent serial trace $t r_{s}^{\prime}$, we remove the operations inserted when constructing $T^{\prime}$ from $T$. This yields traces $t r$ and $t r_{s}$ of $T$. We show in the Appendix that $t r$ is conflictequivalent to $t r_{s}$.

2. We can check view-atomicity of pairs of transactions in $T$ in polynomial time based on Theorem 4.5 . In the following we prove that checking view-atomicity can be reduced to checking conflictatomicity when all pairs of transactions in $T$ are view-atomic. Because all pairs of transactions in $T$ are view-atomic, all nonunit-final writes and non-unit-initial reads do not interact with other transactions, so we remove them. Let $T_{f}$ denote the resulting set of transactions. We prove that $T$ is view-atomic iff $T_{f}$ is conflictatomic. " $\Rightarrow$ ": We prove the contrapositive by constructing a nonview-serializable trace of $T$ from a non-conflict-serializable trace of $T_{f}$. " $\Leftarrow$ ": For each trace $t r$ of $T$, there is a corresponding trace $t r_{f}$ of $T_{f}$ which has a conflict-equivalent serial trace $t r_{f}^{s}$. A serial trace $t r^{s}$ of $T$ that is view-equivalent to $t r$ can be constructed from $t r_{f}^{s}$ by restoring the removed write and reads.

\section{Comparison with Other Atomicity Checking Algorithms}

\subsection{Reduction-Based Algorithms}

Reduction-based algorithms for checking conflict-atomicity [8, 5, 22] classify events based on commutativity and apply Lipton's reduction theorem [15]. We briefly describe the reduction-based algorithm in [22], which is more accurate than the others.

An event is a right-mover $(R)$ if, whenever it appears immediately before an event of a different thread, the two events can be swapped (i.e., they can be executed in the opposite order without blocking) without changing the resulting state. A left-mover $(L)$ is defined similarly. Events not known to be left or right movers are non-movers $(N)$. Race-free events are both right and left movers; events with race are non-movers. Lock acquire events are rightmovers. Lock release events are left-movers. The reduction-based algorithm is based on the following variant of Lipton's reduction theorem: a set $T$ of transactions is conflict-atomic if $T$ has no potential for deadlock and each transaction in $T$ has the form $\left(R+A c q A^{*} \operatorname{Rel}\right)^{*} N^{?}\left(L+A c q A^{*} R e l\right)^{*}$, where $R, L$, and $N$ denote right-mover, left-mover, and non-mover respectively, and $A c q A^{*}$ Rel denotes an acquire of some lock, followed by accesses to read-only or thread-local variables, followed by release of the same lock.

The following theorem and example together show that Theorem 4.1 is more accurate than the above reduction theorem for conflict-atomicity.
Theorem 6.1. If a transaction $t$ has the form

$\left(R+A c q A^{*} \operatorname{Rel}\right)^{*} N^{?}\left(L+A c q A^{*} R e l\right)^{*}$, then $t$ has at most one commit node in the conflict-forest.

Proof. According to the algorithm in Figure 3, the block denoted by $A c q A^{*} R e l$ does not contain any communication node. All synchronization blocks denoted by $R^{*} N^{?} L^{*}$ must be nested. Thus, for any two communication nodes in $t$ that are also synchronization nodes, one is a descendant of the other. $t$ contains at most one non-mover, and it occurs in the inner-most synchronization block. Thus, there is at most one access node in $t$ that is also a communication node, and that communication node is a descendant of all other communication nodes in $t$. Thus, for any two communication nodes of transaction $t$, one is a descendant of the other. Hence, $t$ has at most one commit node.

Now we give an example that is conflict-atomic according to Theorem 4.1 but is wrongly reported to be non-atomic by the reduction theorem. Let $T=\left\{t_{1}, t_{2}\right\}$, where $t_{1}$ consists of $e_{x}^{r_{1}}$ followed by $e_{x}^{w_{1}}$, and $t_{2}$ consists of only $e_{x}^{r_{2}} \cdot t_{1}$ has the form $N N$ which does not match $\left(R+A c q A^{*} R e l\right)^{*} N^{?}\left(L+A c q A^{*} R e l\right)^{*}$, but $t_{1}$ and $t_{2}$ each have only one commit node.

The commit-node algorithm of Section 4.1 contains the benefits of all the improvements to the reduction-based algorithm described in [22], which include the improvements proposed in [5]. For example, for re-entrant locks, thread-local locks, and protected locks, there is no inter-edge connected to the corresponding synchronization nodes.

Therefore, the reduction-based algorithm is less accurate than the commit-node algorithm of Section 4.1.

\subsection{The Block-Based Algorithm}

The block-based algorithm [22] checks view-atomicity by considering pairs of blocks from different transactions. Intuitively, a block captures the information about two accesses and the associated synchronization that is relevant to atomicity checking. The block-based algorithm constructs blocks from an observed trace and then compares each block with all blocks in other concurrent transactions. If two blocks are found to match certain unserializable patterns, the transactions containing them are not atomic. The unserializable patterns are defined based on view serializability; for example, one unserializable pattern is when a write of one transaction can happen between two continuous reads of another concurrent transaction.

The commit-node algorithm and the block-based algorithm are both exact tests for view-atomicity for two transactions, but the former runs much faster in most programs in the experiments in Section 8. For three or more transactions, the commit-node algorithm is an efficient conservative test that is very accurate in practice based on the experiments in Section 8; the block-based algorithm can provide an exact test but is significantly more expensive.

\section{Implementation}

We implemented the commit-node algorithms for checking conflictatomicity and view-atomicity in Java. The implementation consists of three parts: instrumentation, monitoring and off-line analysis. Instrumentation is discussed in Section 7.1. The monitor intercepts all events described in Section 2 and constructs access trees. Each access tree is optimized to discard the redundant accesses, as discussed in Section 7.2. If there are more than two identical access trees, we save only two copies, since the rest are redundant for checking atomicity. A dynamic escape analysis introduced in Section 7.3 is used to determine when a variable escapes. A happen-before analysis introduced in Section 7.4 is used to determine whether two units are concurrent. When the program terminates, the algorithm adds inter-edges between access trees, and then 
checks conflict-atomicity and view-atomicity using the algorithms in Sections 4.1 and 4.2, respectively.

\subsection{Instrumentation}

We modify the pretty-printer in the Kopi [14] compiler to insert instrumentation as it pretty-prints the source code. The instrumentation intercepts the following events: (1) reads and writes to all monitored fields (see below); (2) entering and exiting synchronized blocks, including synchronized methods; (3) entering and exiting methods that are considered as transactions (discussed in Section 2); (4) calls to thread start and join; (5) barrier synchronization.

All non-final fields (with primitive type or reference type) of the specified classes (by default, all classes) are monitored. Accesses to these fields in all methods of all classes are instrumented. Local variables are not monitored, because they are accessed by at most one thread. Our system inserts fields into monitored classes to keep track of shadow information, e.g., whether the object has escaped. There is no way to insert fields into array classes in Java, so we maintain a hash table that maps each array reference $a$ to an array with shadow information for each element of $a$. Monitoring every array element causes large slowdown in some programs, so our system supports "sampling" of arrays, in which only index positions below a user-specified cutoff are monitored.

\subsection{Optimization: Trimming the Access Tree}

It is not necessary to save all accesses to escaped variables. For access nodes with the same parent node, we preserve only the first two read accesses and the first two write accesses (if they exist) to each escaped variable, because the first two reads and writes to $x$ can represent all discarded accesses for checking (conflict or view) atomicity. The resulting trees and forests are said to be trimmed.

Theorem 7.1. For every $\langle T, E\rangle$, and every hypothesis $H$ about the conflict-forest and view-forest in the theorems of Section 4, $H$ holds for the trimmed forest iff it holds for the untrimmed forest.

Proof. 1. Consider reads. Let $R$ be a set of three or more reads to the same variable that share the same parent node. It is easy to see that in both conflict forest and view forest, either all of them are connected to a given write in another unit by inter-edges, or none of them are connected to it. Suppose we evaluate $H$ considering only the first two reads in $R$. It is easy to show that considering additional reads in $R$ either does not generate any additional interedges, or the generated edges do not affect $H$.

2. Consider writes in the conflict-forest. Let $W$ be a set of three or more writes to the same variable that share the same parent node. In the conflict forest, either all of them are connected to a given read or write of another unit by inter-edges, or none of them are connected to it. Similarly as for reads, we can show that considering the third and subsequent writes in $W$ either does not generate any additional inter-edges, or the generated edges do not affect $H$. For the view forest, if $W$ does not contain a unit-final write, then the reasoning is similar to the previous cases; if $W$ contains a unit-final write, it gets removed and the second write in $W$ becomes the unitfinal to that variable; it is easy to verify (for each hypothesis $H$ ) that this does not affect $H$.

\subsection{Dynamic Escape Analysis}

Before an object escapes from the thread that created it, all operations on it can be ignored when checking atomicity. An object $o$ escapes in the following scenarios: (1) $o$ is stored in a static field or a field of an escaped object; (2) $o$ is an instance of a thread and the thread is started; (3) $o$ is referenced by a field of another object $o^{\prime}$, and $o^{\prime}$ escapes (this leads to cascading escape); (4) $o$ is passed as an argument to a native method that may cause it to escape.
To indicate whether an object has escaped, a boolean instance field escaped is added to every instrumented class. Its initial value is false. To detect when objects escape, we instrument all method calls, and all stores to static fields, instance fields, and arrays. When an object escapes, it is marked as escaped by setting its escaped field to true, and all objects to which it refers are marked as escaped (and so on, recursively); Java's reflection mechanism is used to dynamically find those objects. More details appear in [22].

\subsection{Happen-Before Analysis}

The execution of a thread is separated into periods by occurrences of synchronization events. A thread period happens before another thread period if it must end before the other thread period starts.

Our happen-before analysis tracks only happen-before relationships induced by start and join on threads and by barrier synchronization. A barrier is a rendezvous point for a specified number $n$ of threads. Once all $n$ threads reach the barrier, all of them may continue executing. Happen-before relationships induced by wait and notify could also be analyzed; we do not do this because we believe that wait and notify are rarely used to achieve atomicity.

We use a directed graph to represent the happen-before relations between thread periods. There is a path from the node representing $p_{1}$ to the node representing $p_{2}$ iff $p_{1}$ happens before $p_{2}$. More details are in [22].

\section{Experiments}

We perform experiments with 12 programs. They are elevator, tsp, sor, and hedc from [19]; moldyn, montecarlo, and raytracer from the Java Grande Benchmark Suite [12]; StringBuffer, Vector, Hashtable, and Stack from Sun JDK 1.4.2; and jigsaw from W3C [13]. elevator simulates the actions of multiple elevators. tsp solves the travelling salesman problem; we run it on the accompanying data file map14. sor is a scientific computing program which uses barriers rather than locks for synchronization. hedc is a Web crawler that searches astrophysics data on the Web. moldyn, montecarlo, and raytracer are computation-intensive parallel programs that compute molecular dynamics, Monte Carlo simulation, and ray tracing, respectively. jigsaw is a Web server implemented in Java; we instrument only its packages that are related to HTTP service. Table 1 shows the number of lines of code in instrumented classes, i.e., it excludes code in uninstrumented libraries. For all programs that accept the number of threads as an argument, we use three threads. All experiments are done on a Sun Blade 1500 with a $1 \mathrm{GHz}$ UltraSPARC III CPU, 2GB RAM, SunOS 5.8, and JDK 1.4.2.

Table 1 compares the running time, result and storage of the commit-node algorithm for view-atomicity described at the end of Section 4.2 with the off-line reduction-based algorithm [22] and the pairwise block-based algorithm [22]. This version of the blockbased algorithm checks view-atomicity of all pairs of transactions; the full block-based algorithm, which checks whether the entire set of transactions is atomic, would be significantly slower. "LOC" is the lines of code. "Base time" is the running time of the uninstrumented program. "Intrcpt time" is the running time when all events relevant to atomicity checking are intercepted but not processed (an empty method is called). For each algorithm, "time" includes the running time of the instrumented program and the subsequent analysis. "space" is the storage used by each algorithm. The storage of the commit-node algorithm is the sum of the number of nodes and the number of inter-edges (which in these experiments is at most $2 / 3$ of the number of nodes) in the trimmed view-forest. The storage of the reduction-based algorithm is the total size of locksets (which store identifiers of lock objects) and thread-sets (which store identifiers of thread periods) for all escaped variables. The storage of the block-based algorithm is the number of blocks. "re- 


\begin{tabular}{|c|c|c|c|c|c|c|c|c|c|c|c|c|}
\hline \multirow[t]{2}{*}{ Program } & \multirow[t]{2}{*}{ LOC } & \multirow{2}{*}{$\begin{array}{l}\text { Base } \\
\text { time }\end{array}$} & \multirow{2}{*}{$\begin{array}{r}\text { Intrcpt } \\
\text { time }\end{array}$} & \multicolumn{3}{|c|}{ Commit-node Alg } & \multicolumn{3}{|c|}{ Reduction-based Alg } & \multicolumn{3}{|c|}{ Block-based Alg } \\
\hline & & & & time & report & space & time & report & space & time & report & space \\
\hline tsp & 706 & $0.24 \mathrm{~s}$ & $0.4 \mathrm{~s}$ & $40.0 \mathrm{~s}$ & $0-2-0$ & 3015 & $32.5 \mathrm{~s}$ & $0-2-0$ & 530 & $8 \mathrm{~m} 59 \mathrm{~s}$ & $0-2-0$ & 13474 \\
\hline hedc & 2197 & $0.6 \mathrm{~s}$ & $0.82 \mathrm{~s}$ & $1.0 \mathrm{~s}$ & $0-0-0$ & 892 & $1.0 \mathrm{~s}$ & $0-0-1$ & 349 & $2.1 \mathrm{~s}$ & $0-0-0$ & 1085 \\
\hline moldyn & 1265 & $44.03 \mathrm{~s}$ & $24 \mathrm{~m} 34 \mathrm{~s}$ & $34 \mathrm{~m} 26 \mathrm{~s}$ & $0-0-0$ & 3819 & $38 \mathrm{~m} 22.1 \mathrm{~s}$ & $0-0-0$ & 810 & $28 \mathrm{~m} 54.6 \mathrm{~s}$ & $0-0-0$ & 132 \\
\hline montecarlo & 3619 & $15.85 \mathrm{~s}$ & $7 \mathrm{~m} 37 \mathrm{~s}$ & $7 \mathrm{~m} 43 \mathrm{~s}$ & $0-0-0$ & 148 & $8 \mathrm{~m} 10.1$ & $0-0-0$ & 79 & $8 \mathrm{~m} 11.4 \mathrm{~s}$ & $0-0-0$ & 159 \\
\hline raytracer & 1832 & $14.34 \mathrm{~s}$ & $10 \mathrm{~m} 8 \mathrm{~s}$ & $10 \mathrm{~m} 50 \mathrm{~s}$ & $2-0-0$ & 106 & $11 \mathrm{~m} 58.9 \mathrm{~s}$ & $2-0-0$ & 26 & $36 \mathrm{~m} 17.6 \mathrm{~s}$ & $2-0-0$ & 39 \\
\hline StringBuffer & 1255 & - & - & - & $0-1-0$ & - & - & $0-1-0$ & - & - & $0-1-0$ & - \\
\hline Vector & 1020 & - & - & - & $4-4-0$ & - & - & $4-4-10$ & - & - & $4-4-0$ & - \\
\hline Hashtable & 1054 & - & - & - & $0-4-0$ & - & - & 0-4-1 & - & - & $0-4-0$ & - \\
\hline Stack & 119 & - & - & - & $3-4-0$ & - & - & $3-4-12$ & - & - & $3-4-0$ & - \\
\hline
\end{tabular}

Table 1. Performance and Accuracy. The categories of "report" for the three algorithms are bug - benign - false alarm. A dash means that the item is negligible.

port" reflects the warnings issued by each algorithm. We classify warnings issued by each algorithm into three categories:

- Bug: the warning reflects a violation of atomicity that might cause a violation of an application-specific correctness requirement.

- Benign: the warning reflects a violation of atomicity that does not affect the correctness of the application.

- False alarm: the warning does not reflect a violation of atomicity.

Table 1 shows, for each category, the number of methods such that a warning in that category is issued for a transaction that is an execution of that method or part of the code of that method. For the commit-node algorithm, we count based on the transactions that do not satisfy the condition in the hypothesis of Theorem 4.6.

We conclude the following from Table 1. (1) The commitnode algorithm has the same accuracy on these benchmarks as the pairwise block-based algorithm, and they are more accurate than the reduction-based algorithm; specifically, they produce no false alarms, while the reduction-based algorithm produces 25 false alarms in total. Diagnosing a warning as a false alarm can require significant human time and effort, so reducing the number of false alarms is crucial in practice. (2) In the experiments, the pairwise block-based algorithm does not miss any atomicity violations involving three or more transactions (i.e., no such violations are present). (3) The commit-node algorithm is as fast as the reductionbased algorithm (even $0.4 \%$ faster on average), and significantly faster than the block-based algorithm (56\% faster on average).

We also check these programs for conflict-atomicity using the commit-node algorithm presented in Section 4.1. It issues exactly the same warnings (including bugs, benign and false alarms) as the commit-node algorithm for checking view-atomicity. This shows that the reduction-based algorithm issues false alarms because its analysis is imprecise, not because it is checking conflict-atomicity while the other algorithms used for Table 1 check view-atomicity. The commit-node algorithm for conflict-atomicity is slightly faster (5.9\% faster on average) than the commit-node algorithm for viewatomicity, because the former needs less time to construct interedges.

We also test the programs by comparing pair of transactions each time according to Theorems 4.2 and 4.5. Checking pairs of transaction for conflict-atomicity and view-atomicity produces the same result as checking the whole set of transactions.

The bugs in raytracer come from atomicity violations involving the field JGFRayTracerBench.checksum1, which could get an incorrect value, causing the program to report failure. The bug in jigsaw is due to atomicity violations involving the field w3c.tools.resources. store.ResourceStoreManager.loadedStore due to statements loadedStore++ and loadedStore-- without synchronization; as a result, loadedStore may contain an incorrect value. The error in jigsaw described in [20] does not appear in our experiments, because the relevant code was modified in the newer version of jigsaw that we tested. The above atomicity violations involve data races. The errors in Vector and Stack are from atomicity violations involving the field elementCount (discussed in Section 1).

The reduction-based algorithm produces more false alarms than the others. For example, some Collection classes use modCount to count modifications. Thus, when an update method $m_{1}$ executes modCount++ (which is a read followed by a write), and another method $m_{2}$ checks for recent modifications by reading modCount, there is a serializable sequence of events $m_{1}: \operatorname{read}(\operatorname{modCount}) \quad m_{2}: \operatorname{read}(\bmod C o u n t) \quad m_{1}:$ write(modCount). But the benign race on modCount causes the reduction-based algorithm to produce a false alarm here, because $m_{1}$ contains two accesses to modCount that are non-movers. Similar scenarios exist in jigsaw (e.g., on the field alive in the method w3c.util.CachedThread.waitForRunner()) and other programs.

\section{Related Work}

In [21, 22], we proposed the reduction-based and block-based algorithms for runtime atomicity checking. Flanagan and Freund [5] independently proposed a reduction-based algorithm. Our previous experiments showed that the reduction-based algorithm is faster, and the block-based algorithm is more accurate [22]. This paper presents a new algorithm that is as fast as the reduction-based algorithm, is as accurate as the pairwise block-based algorithm, and can detect atomicity violations involving any number of transactions. We explored the use of static analysis to decrease the overhead for the reduction-based algorithm [17] and the block-based algorithm [1]. The similar technique can be used to reduce the overhead of the commit-node algorithm as our future work.

Flanagan et. al. extended their atomicity type system to verify abstract atomicity of programs by analyzing purity [7]. We extended their work to verify atomicity of programs that use nonblocking synchronization [23].

Model checking can also be used to check atomicity [9, 4]. Model checking provides stronger guarantees than runtime mon- 
itoring, because it considers all possible behaviors of a program. Also, many of the supporting analyses, such as dynamic escape analysis, analysis of arrays, deadlock detection, and special treatment of thread-local and read-only variables, etc., can be performed more easily and precisely in a model checker than by program instrumentation [9]. However, model checking is more expensive and is feasible only for programs with relatively small state spaces.

von Praun and Gross [20] present a static analysis to detect violations of method consistency, which is similar to atomicity. Although their static analysis is unsound (in order to reduce the cost and the number of false alarms), it considers the entire program and therefore may be more thorough than runtime analysis in some cases. On the other hand, it produces more false alarms than our commit-node algorithm, based on a comparison of the false alarms in our Table 1 with the false and spurious reports in Table 1 of [20].

Linearizability [11] is a correctness condition for objects which are shared by concurrent processes. Linearizability can be viewed as a special case of strict serializability where transactions are restricted to consist of a single method applied to a single object [11]. Linearizability is defined semantically, i.e., in terms of the specification (correctness requirements) of the object. In contrast, we define atomicity in terms of operations performed by the implementation. Our definition is more restrictive but has the practical benefit of being directly applicable to programs for which formal correctness requirements are unavailable. Vafeiadis et. al. present an approach to use rely-guarantee reasoning to verify linearizability of several algorithms using fine-grain synchronization [18]. The approach is not automatic but provides static guarantees and can analyze fine-grain synchronization for which our algorithms produce false alarms.

\section{Conclusions and Future Work}

This paper defines two kinds of atomicity, conflict-atomicity and view-atomicity. In theory, view-atomicity is more appealing because it is less restrictive, but in our experiments, checking viewatomicity and checking conflict-atomicity give the same results. It is well-known that checking conflict-serializability is in P [3] and checking view-serializability is NP-complete [16]; surprisingly, we show that the problems of checking conflict-atomicity and checking view-atomicity are polynomially reducible to each other.

In our experiments, the commit-node algorithms proposed in this paper are as fast as and significantly more accurate than our previous reduction-based algorithm, and they are as accurate as and significantly faster than our previous pairwise block-based algorithm.

Directions for future work include using static analysis to reduce the overhead of the commit-node algorithms, evaluating them on larger applications, and considering fine-grain synchronization.

\section{References}

[1] R. Agarwal, A. Sasturkar, L. Wang, and S. D. Stoller. Optimized runtime race detection and atomicity checking using partial discovered types. In Proc. 20th IEEE/ACM International Conference on Automated Software Engineering (ASE). ACM Press, Nov. 2005.

[2] R. Agarwal, L. Wang, and S. D. Stoller. Detecting potential deadlocks with static analysis and runtime monitoring. In Proceedings of the Parallel and Distributed Systems: Testing and Debugging (PADTAD) Track of the 2005 IBM Verification Conference. Springer-Verlag, Nov. 2005.

[3] P. A. Bernstein, V. Hadzilacos, and N. Goodman. Concurrency control and recovery in database systems. Addison Wesley, 1987.

[4] C. Flanagan. Verifying commit-atomicity using model-checking. In Proc. 11th Int'l. SPIN Workshop on Model Checking of Software, volume 2989 of LNCS, pages 252-266. Springer-Verlag, 2004.
[5] C. Flanagan and S. N. Freund. Atomizer: A dynamic atomicity checker for multithreaded programs. In Proc. of ACM Symposium on Principles of Programming Languages (POPL), pages 256-267. ACM Press, 2004.

[6] C. Flanagan and S. N. Freund. Type inference against races. In Static Analysis Symposium (SAS), volume 3148 of LNCS. Springer-Verlag, Aug. 2004.

[7] C. Flanagan, S. N. Freund, and S. Qadeer. Exploiting purity for atomicity. IEEE Transactions on Software Engineering, 31(4), Apr. 2005.

[8] C. Flanagan and S. Qadeer. A type and effect system for atomicity. In Proc. ACM SIGPLAN Conference on Programming Language Design and Implementation (PLDI). ACM Press, 2003.

[9] J. Hatcliff, Robby, and M. B. Dwyer. Verifying atomicity specifications for concurrent object-oriented software using model-checking. In Proc. 5th International Conference on Verification, Model Checking and Abstract Interpretation (VMCAI), volume 2937 of LNCS. Springer-Verlag, Jan. 2004.

[10] K. Havelund. Using runtime analysis to guide model checking of Java programs. In Proc. 7th Int'l. SPIN Workshop on Model Checking of Software, volume 1885 of LNCS, pages 245-264. Springer-Verlag, Aug. 2000.

[11] M. P. Herlihy and J. M. Wing. Linearizability: a correctness condition for concurrent objects. ACM Transactions on Programming Languages and Systems, 12(3):463-492, July 1990.

[12] Java Grande Forum. Java Grande Multi-threaded Benchmark Suite. version 1.0. Available from http://www.javagrande.org/.

[13] Jigsaw, version 2.2.4. Available from http://www.w3c.org.

[14] Decision Management Systems GmbH, Kopi compiler. Available from http://www.dms.at/kopi/.

[15] R. J. Lipton. Reduction: A method of proving properties of parallel programs. Communications of the ACM, 18(12):717-721, 1975.

[16] C. H. Papadimitriou. The serializability of concurrent database updates. Journal of the ACM, 26(4):631-653, Oct. 1979.

[17] A. Sasturkar, R. Agarwal, L. Wang, and S. D. Stoller. Automated type-based analysis of data races and atomicity. In Proc. ACM SIGPLAN 2005 Symposium on Principles and Practice of Parallel Programming (PPoPP). ACM Press, June 2005.

[18] V. Vefeiadis, M. Herlihy, T. Hoare, and M. Shapiro. Proving correctness of highly-concurrent linearizable objects. In Proc. ACM SIGPLAN 2006 Symposium on Principles and Practice of Parallel Programming (PPoPP). ACM Press, 2006.

[19] C. von Praun and T. R. Gross. Object race detection. In Proc. 16th ACM Conference on Object-Oriented Programming, Systems, Languages and Applications (OOPSLA), volume 36(11) of SIGPLAN Notices, pages 70-82. ACM Press, Oct. 2001.

[20] C. von Praun and T. R. Gross. Static detection of atomicity violations in object-oriented programs. In Journal of Object Technology, vol.3, no. 6, June 2004 .

[21] L. Wang and S. D. Stoller. Run-time analysis for atomicity. In Third Workshop on Runtime Verification (RV03), volume 89(2) of Electronic Notes in Theoretical Computer Science. Elsevier, 2003.

[22] L. Wang and S. D. Stoller. Runtime analysis of atomicity for multithreaded programs. Technical Report DAR-04-14, SUNY at Stony Brook, Computer Science Dept., July 2004. (revised May 2005). To appear in IEEE Transactions on Software Engineering.

[23] L. Wang and S. D. Stoller. Static analysis for programs with nonblocking synchronization. In Proc. ACM SIGPLAN 2005 Symposium on Principles and Practice of Parallel Programming (PPoPP). ACM Press, June 2005. 


\section{A. Conflict-Atomicity}

Let held-outside $(n)$ denote the locks held by the executing thread just before the thread executes the first event of node $n$. Let heldoutside $\left(n_{1}, n_{2}\right)$ denote the locks acquired before the first event node $n_{1}$ and released after the last event of node $n_{2}$ by the executing thread. Let held-mid $\left(n_{1}, n_{2}\right)$ denote the locks acquired and released between the last event of $n_{1}$ and the first event of $n_{2}$ by the executing thread.

Theorem 4.2: Suppose $\langle T, \emptyset\rangle$ has no potential for deadlock, and $T$ contains only two transactions. $\langle T, \emptyset\rangle$ is conflict-atomic iff each transaction in $T$ has at most one commit node in the conflict-forest.

Proof. " $\Leftarrow$ ": it follows from Theorem 4.1.

" $\Rightarrow$ ": Suppose $T=\left\{t, t^{\prime}\right\}$. We show that $\langle T, \emptyset\rangle$ is not conflictatomic if at least one transaction in $T$ has two or more commit nodes. Without loss of generality, suppose $t$ has at least two commit nodes. Let $n_{1}$ and $n_{2}$ denote two commit nodes of $t$. Thus, $t^{\prime}$ has at least one commit node. Otherwise, $t$ cannot have any commit node. There must be a pair of conflicting events, denoted $e_{1}$ and $e_{1}^{\prime}, e_{1} \in n_{1}$ and $e_{1}^{\prime} \in n_{1}^{\prime}$, where $n_{1}^{\prime}$ is a communication node in $t^{\prime}$, and there is an inter-edge between $n_{1}$ and $n_{1}^{\prime}$. Similarly, there must be another pair of conflicting events, denoted $e_{2}$ and $e_{2}^{\prime}, e_{2} \in n_{2}$ and $e_{2}^{\prime} \in n_{2}^{\prime}$, where $n_{2}^{\prime}$ is a communication node in $t^{\prime}$, and there is an inter-edge between $n_{2}$ and $n_{2}^{\prime}$.

Suppose one of $n_{1}^{\prime}$ and $n_{2}^{\prime}$ contains the other or $n_{1}^{\prime}=n_{2}^{\prime}$. Then there is a trace $t r$ where $n_{1}^{\prime}$ and $n_{2}^{\prime}$ happen between $n_{1}$ and $n_{2}$, because held-outside $\left(n_{i}\right) \cap$ held-outside $\left(n^{\prime}\right)=\emptyset$ for $i=1,2$, where $n^{\prime}$ is the outer of $n_{1}^{\prime}$ and $n_{2}^{\prime}$, or $n^{\prime}=n_{1}^{\prime}$ if $n_{1}^{\prime}=n_{2}^{\prime}$. Thus, $t r$ does not have any conflict-equivalent serial trace.

Suppose $n_{1}^{\prime}$ does not contain $n_{2}^{\prime}$ and vice versa. According to the definition of conflict-forest, we know held-outside $\left(n_{1}\right) \cap$ held-outside $\left(n_{1}^{\prime}\right)=\emptyset$ and held-outside $\left(n_{2}\right) \cap$ held-outside $\left(n_{2}^{\prime}\right)=$ $\emptyset$. Furthermore, because there is no potential for deadlock, held$\operatorname{mid}\left(n_{1}^{\prime}, n_{2}^{\prime}\right) \cap$ held-outside $\left(n_{1}, n_{2}\right)=\emptyset$ or held-mid $\left(n_{1}, n_{2}\right) \cap$ held-outside $\left(n_{1}^{\prime}, n_{2}^{\prime}\right)=\emptyset$. Otherwise, if there exists a lock $l_{1} \in$ held-mid $\left(n_{1}^{\prime}, n_{2}^{\prime}\right) \cap$ held-outside $\left(n_{1}, n_{2}\right)$ and a lock $l_{2} \in$ held$\operatorname{mid}\left(n_{1}, n_{2}\right) \cap$ held-outside $\left(n_{1}^{\prime}, n_{2}^{\prime}\right)$, then $t$ acquires $l_{2}$ while holding $l_{1}$, and $t^{\prime}$ acquires $l_{1}$ while holding $l_{2}$, and there is no outer lock to prevent those acquires from being interleaved, so these would be potential for deadlocks. Thus, there is a trace $t r$ where both $n_{1}^{\prime}$ and $n_{2}^{\prime}$ happen between $n_{1}$ and $n_{2}$, or $n_{1}$ and $n_{2}$ happen between $n_{1}^{\prime}$ and $n_{2}^{\prime}$. As in the previous case, $t r$ does not have any conflictequivalent serial trace.

\section{B. View-Atomicity}

Theorem 4.4: Suppose $\langle T, E\rangle$ has no potential for deadlock, and $E$ does not contain any synchronization operations. If each transaction of $T$ has at most one commit node in the view-forest, then $\langle T, E\rangle$ is view-atomic.

Proof. For any trace $\operatorname{tr}$ of $\langle T, E\rangle$, we prove that it has a viewequivalent serial trace $t r^{\prime}$, which is constructed in the same way as in Theorem 4.1. The definition for the commit point of each transaction is the same as in Theorem 4.1. By the same reasoning as in the proof of Theorem $4.1, t r^{\prime}$ is serial and consistent with the synchronization operations, so $t r^{\prime}$ is a trace for $\langle T, E\rangle$.

1 . We prove that each read has the same write-predecessor in $t r$ and $t r^{\prime}$. Consider an arbitrary read $e_{x}^{r}$ of a unit $u \in T \cup E$. If $e_{x}^{r}$ does not have potential write-predecessors in units other than $u$, the $e_{x}^{r}$ has the same write-predecessor in $t r$ and $t r^{\prime}$ because $t r^{\prime}$ preserves the internal order of events for each unit in $T \cup E$.

Suppose $e_{x}^{r}$ has potential write-predecessors in units other than $u$, consider two potential write-predecessors $e_{x}^{w_{1}}$ and $e_{x}^{w_{2}}$, and suppose without loss of generality that $e_{x}^{w_{1}}$ is the write-predecessor of $e_{x}^{r}$ in $t r$. Note that $e_{x}^{w_{1}}, e_{x}^{w_{2}}$ and $e_{x}^{r}$ cannot all be in the same unit. We consider four cases.

(1) If $e_{x}^{w_{1}}, e_{x}^{w_{2}}$ and $e_{x}^{r}$ belong to different units, then there are communication nodes such that $e_{x}^{r} \in n_{r}^{w_{1}}, e_{x}^{r} \in n_{r}^{w_{2}}, e_{x}^{w_{1}} \in n_{w_{1}}^{r}$, $e_{x}^{w_{1}} \in n_{w_{1}}^{w_{2}}, e_{x}^{w_{2}} \in n_{w_{2}}^{r}, e_{x}^{w_{2}} \in n_{w_{2}}^{w_{1}}$, and such that inter-edges exist between $n_{r}^{w_{1}}$ and $n_{w_{1}}^{r}$, between $n_{r}^{w_{2}}$ and $n_{w_{2}}^{r}$, and between $n_{w_{1}}^{w_{2}}$ and $n_{w_{2}}^{w_{1}}$. The inter-edge implies that $n_{w_{1}}^{w_{2}}$ and $n_{w_{2}}^{w_{1}}$ cannot interleave, so $n_{w_{2}}^{w_{1}}$ happens either before $n_{w_{1}}^{w_{2}}$ or after $n_{r}^{w_{2}}$ in $t r$ (if $n_{w_{2}}^{w_{1}}$ happened between $n_{w_{1}}^{w_{2}}$ and $n_{r}^{w_{2}}$, it would contradict the fact that $e_{x}^{w_{1}}$ is the write-predecessor of $e_{x}^{r}$ in $\left.t r\right)$. Thus, the commit point of $n_{w_{2}}^{w_{1}}$ is either before the commit point of $n_{w_{1}}^{w_{2}}$ or after the commit point of $n_{r}^{w_{2}}$ in $t r$. Therefore, $e_{x}^{w_{2}}$ happens either before $e_{x}^{w_{1}}$ or after $e_{x}^{r}$ in $t r^{\prime}$, so $e_{x}^{r}$ has the same write-predecessor $e_{x}^{w_{1}}$ in both $t r$ and $t r^{\prime}$.

(2). If $e_{x}^{w_{1}}$ and $e_{x}^{w_{2}}$ belong to the same unit, then $e_{x}^{w_{2}}$ must happen either before $e_{x}^{w_{1}}$ or after $e_{x}^{r}$ in $t r$. For the first case, $e_{x}^{w_{2}}$ also happens before $e_{x}^{w_{1}}$ in $t r^{\prime}$. For the second case, there must be communication nodes $n_{w_{2}}^{r}$ and $n_{r}^{w_{2}}$ that contain $e_{x}^{w_{2}}$ and $e_{x}^{r}$, respectively, and $n_{w_{2}}^{r}$ happens after $n_{r}^{w_{2}}$ in $t r$. Thus, $e_{x}^{w_{2}}$ also happens after $e_{x}^{r}$ in $t r$. Therefore, $e_{x}^{r}$ has the same writepredecessor $e_{x}^{w_{1}}$ in $t r$ and $t r^{\prime}$.

(3). If $e_{x}^{w_{1}}$ and $e_{x}^{r}$ belong to the same unit, then $e_{x}^{w_{2}}$ must happen either before $e_{x}^{w_{1}}$ or after $e_{x}^{r}$ in $t r$. For the first case, there must be communication nodes $n_{w_{2}}^{w_{1}}$ and $n_{w_{1}}^{w_{2}}$ that contain $e_{x}^{w_{2}}$ and $e_{x}^{w_{1}}$, respectively, and $n_{w_{2}}^{w_{1}}$ happens before $n_{w_{1}}^{w_{2}}$ in $t r$, so $e_{x}^{w_{2}}$ also happens before $e_{x}^{w_{1}}$ in $t r^{\prime}$. For the second case, $e_{x}^{w_{2}}$ happens after $e_{x}^{r}$ in $t r^{\prime}$ by the same reason. Therefore, $e_{x}^{r}$ has the same writepredecessor $e_{x}^{w_{1}}$ in $t r$ and $t r^{\prime}$.

(4). If $e_{x}^{w_{2}}$ and $e_{x}^{r}$ belong to the same unit, then $e_{x}^{w_{2}}$ must happen before $e_{x}^{w_{1}}$ in $t r$, and there must be communication nodes $n_{w_{2}}^{w_{1}}$ and $n_{w_{1}}^{w_{2}}$ that contain $e_{x}^{w_{2}}$ and $e_{x}^{w_{1}}$, respectively, and $n_{w_{2}}^{w_{1}}$ happens before $n_{w_{1}}^{w_{2}}$ in $t r$. This implies $e_{x}^{w_{2}}$ also happens before $e_{x}^{w_{1}}$ in $t r^{\prime}$. Therefore, $e_{x}^{r}$ has the same write-predecessor $e_{x}^{w_{1}}$ in both $t r$ and $t r^{\prime}$.

2. Now, we prove that $t r$ and $t r^{\prime}$ have the same trace-final write to each variable. Suppose that the trace-final write to a variable $x$ in $t r$ is $e_{x}^{f w}$, and another write $e_{x}^{w}$ to $x$ happens before $e_{x}^{f w}$ in $t r$. We prove that $e_{x}^{w}$ also happens before $e_{x}^{f w}$ in $t r$. Let $e_{x}^{w^{\prime}}$ denote the last write to $x$ in the same unit as $e_{x}^{w}$. Because $e_{x}^{f w}$ is the tracefinal write of $t r, e_{x}^{w^{\prime}}$ happens before $e_{x}^{f w}$ in $t r$. According to the algorithm in Figure 5, there must be two communication nodes $n$ and $n^{\prime}$ such that $e_{x}^{f w} \in n$ and $e_{x}^{w^{\prime}} \in n^{\prime}$. Thus, $n^{\prime}$ must happen before $n$. Hence, $e_{x}^{w^{\prime}}$ happens before $e_{x}^{f w}$ in $t r^{\prime}$. Since $e_{x}^{w}$ can either be $e_{x}^{w^{\prime}}$ or happen before $e_{x}^{w^{\prime}}, e_{x}^{w}$ must happen before $e_{x}^{f w}$.

\section{The Polynomial Equivalence of Conflict- and View-Atomicity}

Lemma C.1. Suppose $\langle T, \emptyset\rangle$ has no potential for deadlock, and $T$ contains only two transactions. $\langle T, \emptyset\rangle$ is not conflict-atomic iff there are at least two inter-edges in the conflict-forest.

Proof. Suppose that the two transactions are $t$ and $t^{\prime}$.

" $\Rightarrow$ ": We prove the contrapositive holds. If there is only one or no inter-edge between $t$ and $t^{\prime}$, then it is easy to show that each of them has at most one commit node, so $T$ is conflict-atomic according to Theorem 4.2.

" $\Leftarrow$ ": Suppose that one inter-edge connects node $n_{1}$ of $t$ and node $n_{1}^{\prime}$ of $t^{\prime}$; another inter-edge connects node $n_{2}$ of $t$ and node $n_{2}^{\prime}$ of $t^{\prime}$. These are two different edges, so $n_{1} \neq n_{2}$ or $n_{1}^{\prime} \neq n_{2}^{\prime}$. If $n_{1}$ or $n_{2}$ is the ancestor of the other, $n_{1}^{\prime}$ and $n_{2}^{\prime}$ cannot be ancestor for each other according to the algorithm in Figure 3 (because the "outmost common lock" condition would imply $n_{1}=n_{2}$ and $n_{1}^{\prime}=n_{2}^{\prime}$ ), so there are at least two commit nodes in $t^{\prime}$, hence $T$ is not conflict-atomic according to Theorem 4.2. Otherwise, $t$ 
contains at least two commit nodes, so $T$ is not conflict-atomic according to Theorem 4.2.

Lemma C.2. Suppose $\langle T, \emptyset\rangle$ has no potential for deadlock, and $T$ contains only two transactions. $\langle T, \emptyset\rangle$ is not view-atomic iff there are at least two inter-edges in the view-forest.

Proof. The proof is similar as the proof for Theorem C.1.

Theorem 5.1: Checking conflict-atomicity of $\langle T, \emptyset\rangle$ and checking view-atomicity of $\langle T, \emptyset\rangle$ are polynomially reducible to each other.

Proof. 1. We first prove that the problem of checking conflictatomicity can be polynomially reducible to the problem of checking view-atomicity. We transform $T$ as follows. Let $l$ be a lock not used in $T$. For each variable $x$, each read $e_{x}^{r}$ is replaced by $e_{l}^{a c q} e_{x}^{r} e_{l}^{r e l}$; and each write $e_{x}^{w}$ is replaced by $e_{l}^{a c q} e_{x}^{r} e_{x}^{w} e_{l}^{r e l}$. Let $T^{\prime}$ denote the resulting set of transactions.

Now we prove that $T$ is conflict-atomic iff $T^{\prime}$ is view-atomic.

" $\Rightarrow$ ": For any trace $t r$ of $T, T$ is conflict-atomic implies that $t r$ has an conflict-equivalent serial trace $t r_{s}$, We transform $t r$ and $t r_{s}$ in the same manner that is used to transform $T$, yielding $t r^{\prime}$ and $t r_{s}^{\prime}$ of $T^{\prime}$, respectively. For each read in the original trace $t r$, the corresponding read in $t r^{\prime}$ has the same write-predecessor in $t r^{\prime}$ and $t r$ (because the insertions do not affect this relationship) and the same write-predecessor in $t r$ and $t r_{s}$ (because they are conflict equivalent), and the same write-predecessor in $t r_{s}$ and $t r_{s}^{\prime}$ (because the insertions do not affect this), so it has the same writepredecessor in $t r^{\prime}$ and $t r_{s}^{\prime}$. Similarly, we can show that the final write to each variable is the same in $t r^{\prime}$ and $t r_{s}^{\prime}$. For each read inserted next to a write event, that write has the same order with respect to all other writes in $t r$ and $t r_{s}$, so the associated read has the same write-predecessor in $t r^{\prime}$ and $t r_{s}^{\prime}$. Thus $t r^{\prime}$ has a viewequivalent serial trace $t r_{s}^{\prime}$. Because the lock $l$ is not used in $T$, and is added in the pattern described above, there is a one-to-one correspondence between traces of $T$ and traces of $T^{\prime}$. According to the previous analysis, each trace of $T^{\prime}$ has an view-equivalent serial trace, so $T^{\prime}$ is view-atomic.

" $\Leftarrow$ ": For each trace $t r^{\prime}$ of $T^{\prime}$, we remove the operations inserted when constructing $T^{\prime}$ from $T$. This yields a trace $t r$ of $T$. By assumption $t r^{\prime}$ has a view-equivalent trace $t r_{s}^{\prime}$. Writes to the same variable must occur in the same order in $t r^{\prime}$ and $t r_{s}^{\prime}$. Otherwise, the inserted read next to some write would have a different write predecessor in $t r^{\prime}$ and $t r_{s}^{\prime}$. Using this observation, and similar reasoning as above, we can show that view-atomicity of $T^{\prime}$ implies conflict-atomicity of $T$.

2. Now we prove that the problem of checking view-atomicity is polynomially reducible to the problem of checking conflictatomicity. We can check view-atomicity of pairs of transactions in $T$ in polynomial time based on Theorem 4.5. In the following, we prove that checking view-atomicity can be reduced to checking conflict-atomicity when all pairs of transactions in $T$ are viewatomic.

Because all pairs of transactions of $T$ are view-atomic, all unitnon-final writes cannot be read by other transaction, and each read either reads a preceding write of its own transaction in all traces, or reads writes of other transactions in all traces. We remove from each transaction all unit-non-final writes and all unit-non-initial reads, i.e., all unit-final writes and unit-initial reads are retained. Let $T_{f}$ denote the resulting set of transactions.

Now we prove that $T$ is view-atomic iff $T_{f}$ is conflict-atomic.

" $\Rightarrow$ ": We prove the contrapositive by showing: if $T_{f}$ has a trace $t r$ that is not conflict-serializable, then $t r$ is not view-serializable. Restoring the writes and reads removed when constructing $T_{f}$ does not affect view serializability of the trace $t r$, because those writes are not read by other transactions, and those reads do not read other transactions. Therefore, the resulting trace is a non-viewserializable trace for $T$, so $T$ is not view-atomic.

Let $g$ be the serialization graph for $t r$. Since $t r$ is not conflictserializable, $g$ contains a cycle $c$ of length two or more, i.e., $c$ contains two or more transactions. In the following, we prove by contradiction that $c$ cannot contain exactly two transactions. Suppose that $c$ contains exactly two transactions $t_{j}$ and $t_{j}$, thus, $c$ contains exactly two edges. We first show that the two edges indicate two edges in the conflict-forest for $t_{i}$ and $t_{j}$ which are also in the view-forest for $t_{i}$ and $t_{j}$. For the edge $t_{i} \rightarrow t_{j}$, there must be two conflicting events $e_{i}$ and $e_{j}$ from $t_{i}$ and $t_{j}$, respectively, and $e_{i}$ happens before $e_{j}$ in $t r$; similarly, for the edge $t_{j} \rightarrow t_{i}$, there must be two conflicting events $e_{j}^{\prime}$ and $e_{i}^{\prime}$ from $t_{j}$ and $t_{i}$, respectively, and $e_{j}^{\prime}$ happens before $e_{i}^{\prime}$ in $t r$. There should be at least two inter-edges in the conflict-forest for $t_{i}$ and $t_{j}$. Otherwise, i.e., if there is only one inter-edge between $t_{i}$ and $t_{j}$ (it is easy to know there must be inter-edge(s) because of these conflict events), both $e_{i}$ and $e_{i}^{\prime}$ happen either before or after both $e_{j}$ and $e_{j}^{\prime}$ according to the algorithm in Figure 3. This contradicts with the conclusion discussed above. Therefore, there must be at least two inter-edges in the conflict-forest for $t_{i}$ and $t_{j}$. Because all non-final writes and the following reads are removed, each inter-edge in the conflictforest must be one of the following two kinds of inter-edges:(i) between a unit-initial read of one transaction and a unit-final write to the same variable of the other transaction; or (ii) between a unitfinal write of one transaction and a unit-final write to the same variable of the other transaction. These two kinds of inter-edges also exist in the view-forest, i.e., all edges in the conflict-forest also exist in the view-forest that consists of the same transactions. Thus, if $c$ contains only two transactions, the view-forest would contain two inter-edges between nodes in those two transactions, so according to Lemma C.2, the two transactions would not be view-atomic; this contradicts with the assumption that all pairs of transactions are view-atomic. Hence $c$ contains at least three transactions. Let $T^{\prime}$ denote the set of transactions contained on $c$. Hereafter we focus on the transactions in $T^{\prime}$. Let $t r^{\prime}$ denote the subsequence of $t r$ obtained by removing all events of transactions not in $T^{\prime}$.

The algorithm in Figure 8 shows an algorithm to generate a shortened cycle $c_{v}$ from $c$. We will show that existence of this cycle implies $T$ is not view-atomic. The first three cases identify and mark the edges that denote precedence between transactions for all serial traces view-equivalent to $t r$, i.e., if an edge $t_{1} \rightarrow t_{2}$ is marked, then $t_{1}$ precedes $t_{2}$ in all serial traces view-equivalent to $t r$. The next two cases add shortcut edges to shorten $c$; the new shortcut edges are immediately marked because they also denote precedence between transactions for all serial traces viewequivalent $t r$. In the algorithm, $c$ is updated in each iteration, and $t r^{\prime}$ is updated accordingly by removing the transactions not on the current $c$. Let $c_{v}$ and $t r_{v}$ denote the cycle $c$ and trace $t r^{\prime}$ when the algorithm terminates. Note that $t r_{v}$ is a sub-sequence of the original $t r^{\prime} . t r_{v}$ does not have any view-equivalent serial trace, because of the cyclic precedence of transactions indicated by $c_{v}$. More details are discussed next.

Each edge on $c$ must imply one of the six cases shown in the cases view write-read, view trace_initial_read-write, view writetrace_final_write, write-read, write1-write2, and read-write. The added edge $e g^{\prime}$ in each case also belongs to one of the six cases.

An edge from $t_{i}$ to $t_{j}$ marked in the case by "view write-read" implies that $t_{i}$ contains a write $e_{x}^{w}$ whose written value is read by $t_{j}$. Thus, for any sub-trace of $t r^{\prime}$ obtained by deleting transactions other than $t_{i}$ and $t_{j}$, if it contains the write and read to $x$ in $t_{i}$ and $t_{j}$, respectively, $t_{i}$ precedes $t_{j}$ in all serial traces view-equivalent 
to that sub-trace. Edges marked in the cases view trace_initial_readwrite and view write-unit_final_write have the similar implication.

In the case write-read, the write-predecessor of the read must be in a third transaction, i.e., $t_{k}$ is neither $t_{i}$ nor $t_{j}$. To see this, first note that the write-predecessor of $e_{x}^{r}$ cannot be $e_{x}^{r}$, otherwise this edge would be handled in the case "view write-read". Therefore, if $t_{k}$ were $t_{i}, t_{i}$ would contain two writes to $x$ contradicting the fact that each transaction in $T_{f}$ contains at most one write to each variable. If $t_{k}$ were $t_{j}, e_{x}^{r}$ would not be a unit-initial read in $t_{j}$, contradicting the fact that unit-non-initial reads are removed when constructing $T_{f}$. In the following, we show by contradiction that $c$ contains at least three transactions after being shortened. Suppose $c$ contains only two transactions, namely $t_{k}$ and $t_{j}$, after being shortened. $c$ contains an edge $t_{i} \rightarrow t_{k}$, which was on $c$ already, and the new-edge $t_{k} \rightarrow t_{j}$. From the definition of view-forest, the view-forest contains inter-edges corresponding to these two edges, so according to Lemma C. $2, t_{j}$ and $t_{k}$ are not view-atomic; this contradicts the assumption. The algorithm marks the edge $t_{k} \rightarrow t_{j}$ because $t_{k}$ must precede $t_{j}$ in all serial traces view-equivalent to sub-traces of $t r^{\prime}$ that contain $t_{k}$ and $t_{j}$.

In the case write1-write2, the trace-final write must be in a third transaction. If $t_{k}$ were $t_{i}, t_{i}$ would contain two writes to the same variable, which implies a contradiction. If $t_{k}$ were $t_{j}$ this edge would be processed in the case view write-unit_final_write instead. After being shortened in this case, $c$ must contain at least three transactions. Otherwise, suppose $c$ contains only two transactions, namely $t_{i}$ and $t_{k}$, by the similar reasoning as above, the shortened cycle $c$ contains at least three transactions after this step. The added edge $e g^{\prime}$ always implies that $t_{i}$ must happen before $t_{k}$ in all serial traces view-equivalent to sub-traces of $t r^{\prime}$ that contain $t_{i}$ and $t_{k}$.

In the case "read-write", $t_{k}$ cannot be $t_{i}$ because the $e_{x}^{r}$ is a unitinitial read. $t_{k}$ cannot be $t_{j}$ because each transaction cannot have two writes to the same variable. $t_{h}$ cannot be $t_{i}$ because $e_{x}^{r}$ is a unit-initial read. Note that $t_{h}$ may be $t_{j}$. Similar as before, $c$ must contains at least three transactions after this step of shortening cycle by the similar reasoning as above.

After each iteration of the algorithm in Figure 8,c is always a cycle with at least three nodes (i.e., transactions), hence, $c_{v}$ is a cycle and contains at least three transactions. Each edge $t_{i} \rightarrow t_{j}$ of $c_{v}$, implies that transaction $t_{i}$ must happen before $t_{j}$ in all serial traces view-equivalent to $t r_{v}$. Thus, existence of cycle $c_{v}$ implies that there is no serial trace view-equivalent to $t r_{v}$. If we consider only the transactions contained by $t r_{v}$, after restoring the deleted writes and reads, the resulting trace is still not view-serializable because these deleted writes and reads do not interact with any transaction except for its own. Because the set of transactions in this trace is a subset of $T, T$ is not view-atomic.

" $\Leftarrow$ ": Suppose $T_{f}$ is conflict-atomic. This implies $T_{f}$ is also view-atomic. For each trace $\operatorname{tr}$ of $T$, there is a corresponding trace $t r_{f}$ of $T_{f}$. Since $T_{f}$ is view-atomic, there is a serial trace $t r_{f}^{s}$ that is view-equivalent to $t r_{f}$. We can expand $t r_{f}^{s}$ into a serial trace $t r^{s}$ for $T$ by restoring the removed unit-non-initial reads and unitnon-final writes for each transaction. $t r^{s}$ is view-equivalent to $t r$ because all reads have the same write-predecessor in $t r$ and $t r^{s}$, and all trace-final writes are the same in $t r$ and $t r^{s}$. Hence, $T$ is view-atomic.

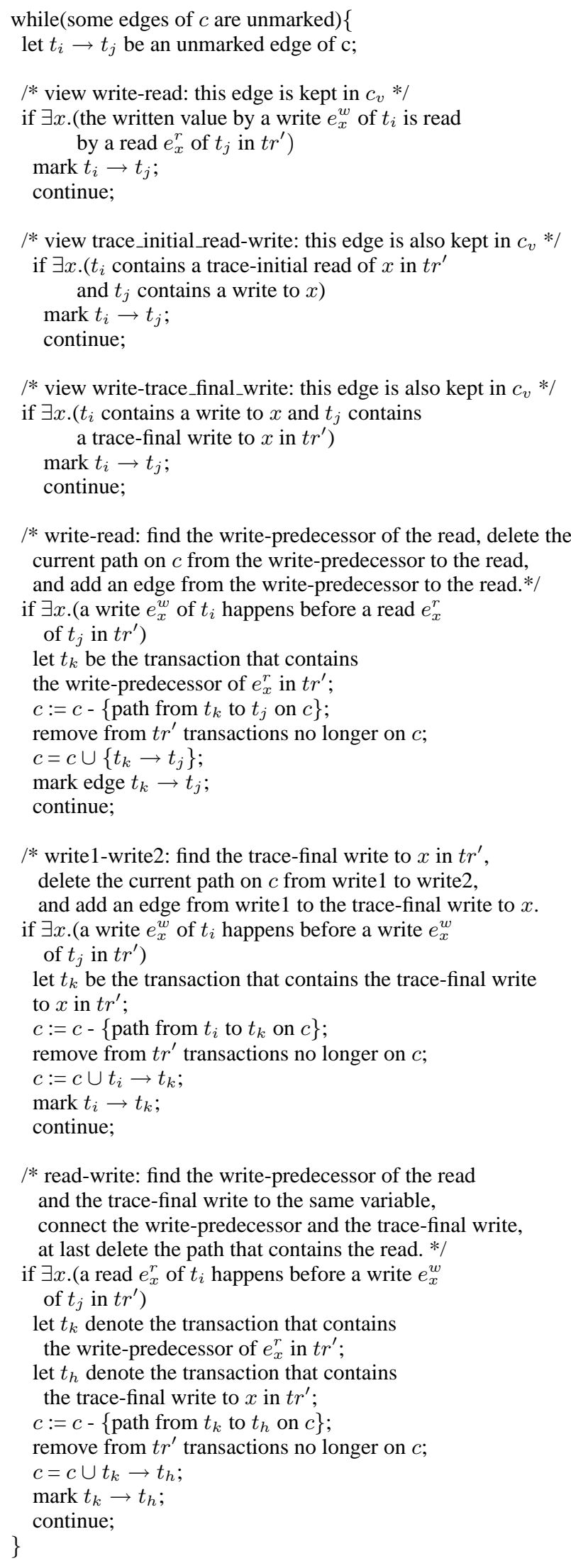

Figure 8. The algorithm to reduce a conflict-atomicity violating cycle to a view-atomicity violating cycle. 